\title{
PROstate Multicentre External beam radioTHErapy Using a Stereotactic boost: the PROMETHEUS study protocol
}

\author{
Matthew Richardson ${ }^{1}$, Mark Sidhom², Sarah Gallagher ${ }^{1}$, Mel Grand ${ }^{2,3}$, David Pryor ${ }^{4}$, Joseph Bucci ${ }^{5}$, Lee Wilton ${ }^{1}$, \\ Sankar Arumugam², Sarah Keats ${ }^{2}$ and Jarad M. Martin ${ }^{1 *}$
}

\begin{abstract}
Background: High Dose Rate Brachytherapy (HDRB) boost is a well-established treatment for prostate cancer (PC). We describe the PROstate Multicentre External beam radioTHErapy Using Stereotactic boost (PROMETHEUS) study. Non-surgical stereotactic techniques are used to deliver similar doses to HDRB boost regimens with a dose escalation sub-study.

Methods: Eligible patients have intermediate or high risk PC. PROMETHEUS explores the safety, efficacy and feasibility of multiple Australian centres cooperating in the delivery of Prostate Stereotactic Body Radiotherapy (SBRT) technology. A SBRT boost component Target Dose (TD) of 19Gy in two fractions is to be delivered, followed by a subsequent EBRT component of 46Gy in 23 fractions. Once accrual triggers have been met, SBRT doses can be escalated in $1 \mathrm{~Gy}$ increments to a maximum of 22Gy in two fractions. Patient safety will also be measured with the rate of both acute and late moderate to severe Gastro-Intestinal (GI) and Genito-Urinary (GU) Common Terminology Criteria for Adverse Events (CTCAE) toxicities as well as patient reported quality of life. Efficacy will be assessed via biochemical control after 3 years.
\end{abstract}

Discussion: PROMETHEUS aims to generate evidence for a non-surgical possible future alternative to HDRB boost regimens, and introduce advanced radiotherapy techniques across multiple Australian cancer centres.

Trial registration: The study was retrospectively registered on the ANZCTR (Australian New Zealand Clinical Trials Registry) with trial ID: ACTRN12615000223538.

Keywords: Radiation oncology, Prostatic neoplasms, Dose Hypofractionation, Radiosurgery, Planning techniques

\section{Background}

Prostate Cancer (PC) is a common malignancy in Australian men. In men with localized disease, external beam radiotherapy (EBRT) is a regularly used management option.

Conventional fraction sizes of 1.8-2 Gy per day are considered to be a standard treatment approach. There is, however, a growing body of evidence to support the efficacy and safety of larger doses per fraction [1-3]. This stems from clinical radiobiological data suggesting that $\mathrm{PC}$ has a low alpha/beta ratio (ABR), and as such a therapeutic ratio could be exploited between the differential

\footnotetext{
* Correspondence: Jarad.martin@calvarymater.org.au

${ }^{1}$ Calvary Mater Newcastle, Newcastle, NSW, Australia

Full list of author information is available at the end of the article
}

fraction size sensitivity of the prostate and the adjacent critical structures, especially the rectum $[4,5]$.

A fundamental concept in radiation oncology is that Tumour Control Probability (TCP) increases as a function of radiation dose. This principle has been validated in several randomized trials of EBRT, with all studies demonstrating an improvement in Prostate Specific Antigen (PSA) control Biological Non-Evidence of disease (bNED) [6, 7]. However, this increased efficacy came at the cost of increased late rectal toxicity [8], and has spurred the development of new technologies to escalate dose to the prostate, while sparing neighbouring critical structures.

Brachytherapy has a history spanning over two decades as one method of escalating dose to the prostate.

(C) The Author(s). 2018 Open Access This article is distributed under the terms of the Creative Commons Attribution 4.0 International License (http://creativecommons.org/licenses/by/4.0/), which permits unrestricted use, distribution, and 
Being surgically implanted into the prostate, brachytherapy is an extremely conformal treatment approach, allowing radiotherapy dose to be tailored very precisely to the required volume. Either Low Dose Rate Brachytherapy (LDRB) or High Dose Rate Brachytherapy (HDRB) can also be combined with $45-50$ Gy of conventionally fractionated EBRT as a dose escalation strategy. Data has been published from several Australian centres confirming the feasibility, efficacy, and low rectal toxicity associated with a HDRB approach $[9,10]$.

Despite this evidence, brachytherapy dose escalation is only available in a relatively limited number of cancer hospitals in Australia. This is mainly due to the need for specialised personnel, theatre access, concerns regarding urethral stricture rates and the high cost of maintaining a HDRB unit [9].

All radiotherapy centres in Australia have the capacity to deliver escalated doses of radiotherapy of 76 Gy or higher. To do this safely, newer technologies have been widely introduced over the last decade. Three Dimensional (3D) planning techniques permit more detailed anatomical information to be incorporated into the design of a patient's treatment. Image Guided Radiotherapy (IGRT) allows more precise delivery of radiation dose to the prostate [11]. Intensity Modulated Radiotherapy (IMRT) using dynamic beam shaping and inverse planning approaches facilitates shaping the higher doses of radiation to irregular shapes [12]. The combination of all of these techniques has led to reduced rates of late grade 2-3 rectal toxicity in the dose escalated setting from $26 \%$ reported in the most mature of the randomized studies [6] to $\sim 6 \%$ in single institution reports [13]. As a result, these approaches feature in current Australasian guidelines and are now widely practiced in Australia [4].

Despite recent advances, it is likely that we are reaching a threshold effect with EBRT alone.

The highest reported conventional doses delivered are 86.4 Gy in 1.8 Gy fractions from MSKCC [14]. Retrospective comparison of this regimen with brachytherapy boost alternatives showed markedly inferior biochemical and metastatic disease control [15]. Advances in EBRT including IMRT and IGRT, as well as emerging evidence for hypofractionation in PC have opened up an avenue to explore Stereotactic Body Radiotherapy (SBRT) as an alternative to HDRB as a prostate radiation dose escalation strategy.

\section{Methods/Design}

PROMETHEUS is a Phase 2 multicentre clinical trial exploring a stereotactic Radiotherapy Boost to the prostate with fractionated external beam radiotherapy. We aim to test the following hypotheses; (1) That radiotherapy dose escalation to the prostate via a SBRT boost is safe using a linear accelerator in the multi-centre setting. (2) That radiotherapy dose escalation to the prostate via a SBRT boost can be increased in a stepwise manner and finally, (3) that radiotherapy dose escalation to the prostate via a SBRT boost is feasible using a Linear Accelerator in the multi-centre setting. The study design is shown in Fig. 1.

\section{Key selection criteria \\ Inclusion criteria}

Men capable of giving informed consent with a histological diagnosis of intermediate or high risk prostate adenocarcinoma as defined by any one of: a) Baseline PSA 10-20, Gleason grade 7 disease, Clinical stage T2b-c OR b) Baseline PSA $\geq 20$ Gleason grade 8-10 disease, Clinical stage T3. Once deemed eligible, recommended 6 months Androgen Deprivation Therapy (ADT) for unfavourable intermediate risk or low-high risk men (1 high risk factor), and 18-24 months for men with multiple high risk factors.

\section{Exclusion criteria}

Patients having received previous pelvis radiotherapy, ECOG performance status $>1$, hip prosthesis in-situ, inability to have a Magnetic Resonance Imaging (MRI), clinical stage T4 disease, presence of inflammatory bowel disease or severe obstructive urinary symptoms, and finally an inability to meet planning objectives.

\section{Objectives}

The primary objectives of the study are to determine the safety and efficacy of a SBRT prostate boost. Safety will be established if the cumulative rate of either acute or late GI or GU toxicity is equivalent to or less than previously reported in the HDRB or dose escalated IGRT EBRT literatures. Efficacy will be assessed via biochemical control after 3 years.

The secondary objective of the study is to ensure that the rates of grade 3 or higher acute CTCAE GI or GU toxicity does not increase with increasing dose delivery to the Clinical Target Volume (CTV).

Multicentre feasibility will be demonstrated if 3 or more centres contribute 5 or more patients to the study.

\section{Treatment planning}

A Rectal Displacement Device (RDD) is to be used with the main options being the transperineal application of SpaceOAR, or transanal insertion of a Rectafix ${ }^{\mathrm{mat}}$ to increase distance from rectum to prostate $[16,17]$.

\section{Simulation}

Intraprostatic fiducial markers will be inserted at least 7 days prior to treatment planning. An appropriate 


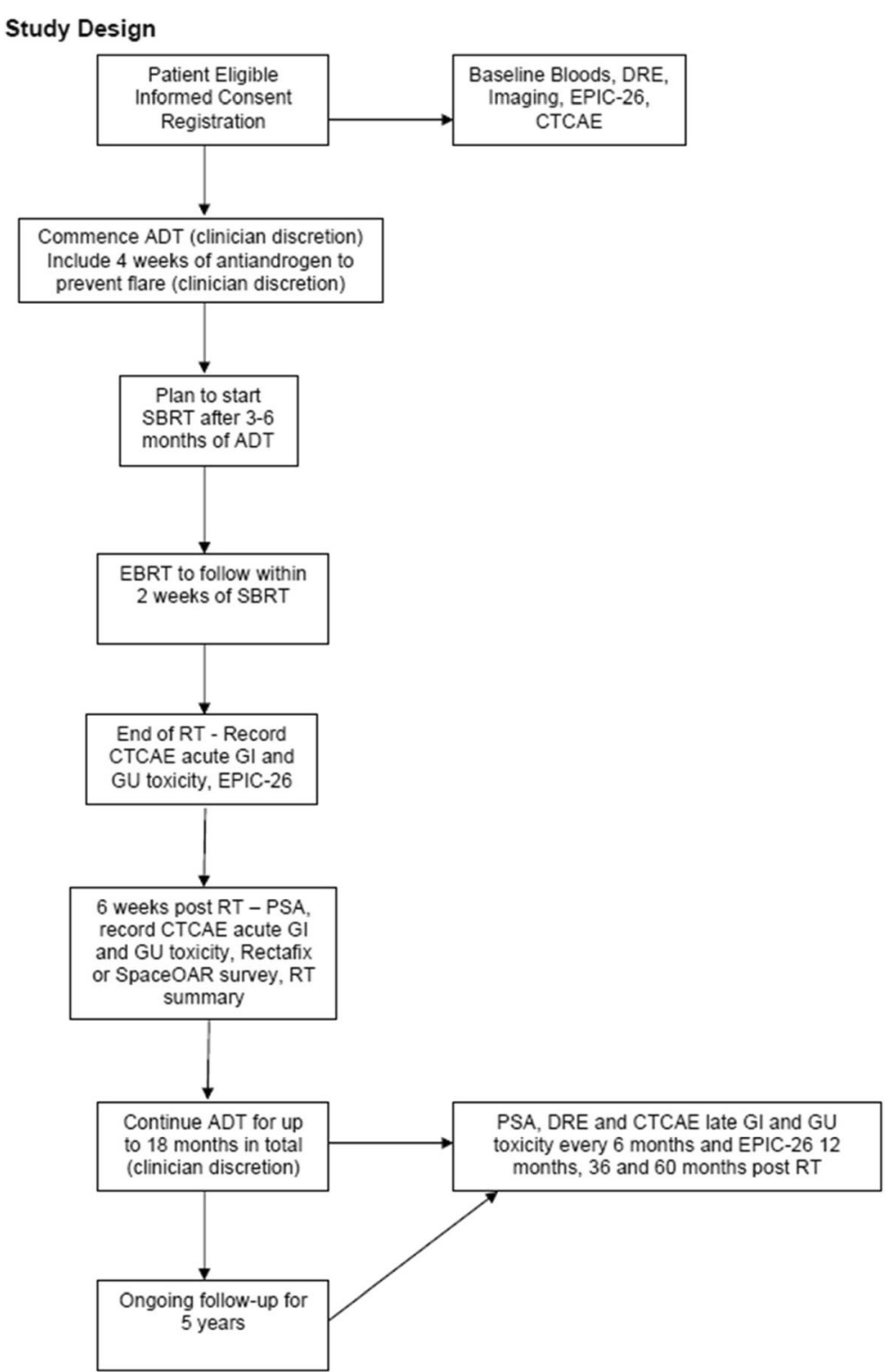

Fig. 1 PROMETHEUS trial study design

RDD is selected for rectal separation. A temporary In-Dwelling urinary Catheter (IDC) can be inserted for planning scans to aid urethral contouring at clinician's discretion. Patients will be instructed to follow bladder and bowel preparation to achieve an empty rectum and full bladder.

A planning Computed Tomography (CT) scan is acquired in both SBRT and EBRT treatment positions. Minimum CT slice thickness of $2.5 \mathrm{~mm}$, scanning from L4 to include whole pelvis to below the perineum. A SBRT planning MRI with RDD in-situ is to be performed within $1 \mathrm{~h}$ of CT to minimize effect of prostate deformation caused by variable bowel filling.

\section{SBRT treatment planning}

CT-MRI fusion of planning scans using prostatic fiducial registration is performed.

The CTVsbrt is the prostate plus any observed extraprostatic disease, either due to T3a extracapsular extension (ECE) or seminal vesicle invasion (SVI). For SVI include disease observed on MRI only for the boost. The SBRT Planning Target Volume (PTVsbrt) expansion is $5 \mathrm{~mm}$ in all directions from CTVsbrt, except posteriorly where it is $3 \mathrm{~mm}$.

Table 1 lists the critical structure contouring guidelines.

Inverse planned IMRT or related modulated dose techniques (Tomotherapy, Cyberknife [CK] or Volumetric 
Table 1 Critical structure contouring guidelines

\begin{tabular}{|c|c|}
\hline Structure name & Description \\
\hline Rectal Wall & $\begin{array}{l}\text { Contour as a } 3 \mathrm{~mm} \text { thick wall structure } \\
\text { from recto-sigmoid junction to lower } \\
\text { aspect of ischial tuberosities. }\end{array}$ \\
\hline Rectal Mucosa & $\begin{array}{l}\text { Solid structure corresponding to the } \\
\text { internal cylindrical space central to the } \\
\text { inner surface of the rectal wall. }\end{array}$ \\
\hline Rectum Posterior Wall & $\begin{array}{l}\text { This is the most posterior } 15 \mathrm{~mm} \text { of } \\
\text { rectal wall }\end{array}$ \\
\hline Bladder & $\begin{array}{l}\text { Contour the whole organ as a solid } \\
\text { structure. }\end{array}$ \\
\hline Penile Bulb & Contour from MRI. \\
\hline $\begin{array}{l}\text { Prostatic urethra Planning } \\
\text { Target at Risk Volume (PRV) }\end{array}$ & $\begin{array}{l}\text { Contour urinary IDC within prostate, and } \\
\text { add } 1 \mathrm{~mm} \text { radial expansion for the PRV. } \\
\text { If no IDC used, estimate urethral } \\
\text { position, and add } 3 \mathrm{~mm} \text { radial expansion. }\end{array}$ \\
\hline Neck of Femur & $\begin{array}{l}\text { Contour the Left and Right NOF as solid } \\
\text { structures to the level of the ischial } \\
\text { tuberosity. }\end{array}$ \\
\hline
\end{tabular}

Modulated Arc Radiotherapy [VMAT]) are required. For static field IMRT, between 5 and 8 fields recommended. Photon energies of 6-10 MV, with higher energies not recommended due to the higher potential for neutron scatter with the large daily doses. An exception is patients with large separations where 1-2 higher energy beams may be helpful in improving dose distribution, but only if all other beams are 6-10 MV. Non-coplanar beams are allowed. VMAT may provide the advantage of more rapid treatment time reducing the need for interim imaging to manage intrafraction motion.

SBRT dose constraints should be met as per Table 2:

Aim to encompass the entire PTVsbrt with 19Gy minimum. However, PTVsbrt coverage may be compromised posteriorly to a minimum of $16 \mathrm{~Gy}$ only where necessary in order to meet rectal dose constraints. Aim for 16Gy isodose line to only encompass posterior PTVsbrt where there is overlap between PTVsbrt and Rectum (see Figs. 2 and 3).

The mechanism for the stepwise SBRT dose escalation is as follows:

A minimum of 20 men in total are to complete treatment without $>15 \%$ suffering a grade 3 acute toxicity or any episodes of grade 4 acute toxicity at a particular dose level. The individual centre must also accrue $\geq 5$ patients at the previous dose level prior to exploring dose escalation. Once this accrual trigger has been met, increase the dose by 1 Gy to D98\% of the volume CTVsbrt minus urethral PRV. The first dose escalation will therefore be to $20 \mathrm{~Gy}$. This process can be followed a maximum of three times, to a maximum CTVsbrt-Urethral PRV D98 of 22 Gy.

A credentialing "dummy run" will need to be performed on a previous patient data set to demonstrate to an external reviewer the feasibility of achieving these new dose constraints. The first three patients planned at each dose level will be subject to a real-time QA process. An external RO review of target delineation, OAR doses and plan quality shall be undertaken before any planned treatment is delivered.

\section{EBRT treatment planning}

The EBRT component of the treatment package will follow the SBRT. CTVebrt will be at the discretion of the treating clinician. For ECE, a $3 \mathrm{~mm}$ margin around the prostate is recommended, excluding the rectal wall. For SVI if no gross T3b disease is detected, the proximal $20 \mathrm{~mm}$ of the seminal vesicle should be included. For Lymph-node invasion (LNI), the pelvic nodal RT is recommended if the LNI risk is $>15 \%$ as indicated by the MSKCC Prostate Nomogram. If treated, pelvic nodes shall be contoured as per the Radiation Therapy Oncology Group (RTOG) guidelines with a superior limit of the bifurcation of the common iliac arteries, and excluding the pre-sacral nodes as reflected by current surgical recommendations of the extent of an extended lymph node dissection [18]. CTVebrt to PTVebrt expansion will be $5-7 \mathrm{~mm}$, or $7 \mathrm{~mm}$ if gross SVI is present around this structure in particular.

46 Gy in 23 fractions will be prescribed to $95 \%$ of the PTVebrt as per ICRU 83. An IMRT, VMAT or Tomotherapy technique is recommended. Dose constraints are outlined in the accompanying Table 3.

\section{Treatment delivery}

Due to potential volume changes during a course of EBRT, as well as evidence of volume changes after HDRB, all patients should receive the boost component of their therapy prior to the fractionated EBRT component [19, 20].

The two SBRT boost fractions are to be separated by a 1 week break. The EBRT component is then to commence 2 weeks after the final boost fraction.

Preparation: Patient to continue same bladder preparation and enema regimen as used during simulation. Use Rectafix $^{\mathrm{TM}}$ if utilised during simulation.

IGRT: On-line correction to gold fiducials, prostate and IDC with $0 \mathrm{~mm}$ action threshold. All effort should be made to commence treatment as soon as feasible after pre-treatment imaging has been performed.

During SBRT treatment: For techniques where real-time tracking or position monitoring is available (e.g. CK kV or intraprostatic transponders) this should be used. For other approaches, aim to perform repeat orthogonal imaging at least every $4 \mathrm{~min}$, as there is evidence to support that $95 \%$ of patients will experience less than $3 \mathrm{~mm}$ of intrafraction motion over this timeframe [21]. Since most intrafraction motion will be 
Table 2 SBRT dose constraints. (TD = Target dose)

\begin{tabular}{|c|c|c|c|}
\hline Constraint & Per-Protocol & Minor Variation & Major Variation \\
\hline CTVsbrt D98 ${ }^{\text {a }}$ & $>100 \% \mathrm{TD}$ & $95-100 \%$ TD & $<95 \%$ TD \\
\hline PTVsbrt D50 a & $<105 \%$ TD & $105-110 \%$ TD & $>110 \% \mathrm{TD}$ \\
\hline PTVsbrt D90 a & $>100 \%$ TD & $95-100 \%$ TD & $<95 \%$ TD \\
\hline PTVsbrt D95 $5^{\mathrm{a}}$ & $>95 \% \mathrm{TD}$ & $90-95 \%$ TD & $<90 \%$ TD \\
\hline PTVsbrt D99 & $>16 \mathrm{~Gy}$ & 15-16 Gy & $<15$ Gy \\
\hline PTVsbrt Dmax to $0.1 \mathrm{cc}$ & $<110 \%$ TD & $110-120 \%$ TD & $>120 \% \mathrm{TD}$ \\
\hline PTVsbrt Dmax & \multicolumn{3}{|c|}{ Not within a critical structure } \\
\hline Rectal Wall Dmax to $0.1 c c$ & $<17 \mathrm{~Gy}$ & 17-17.5 Gy & $>17.5 \mathrm{~Gy}$ \\
\hline Rectal Wall V16 Gy & $<0.5 c c$ & $0.5-1 c c$ & $>1 c c$ \\
\hline Rectal Wall V14 Gy & $<3 c c$ & $3-5 c c$ & $>5 c c$ \\
\hline Rectal Wall V12 Gy & $<30 \%$ & $30-40 \%$ & $>40 \%$ \\
\hline Rectal Wall V10 Gy & $<40 \%$ & $40-50 \%$ & $>50 \%$ \\
\hline Rectal Wall V8 Gy & $<60 \%$ & $60-70 \%$ & $>70 \%$ \\
\hline Rectal Mucosa Dmax to $0.1 \mathrm{cc}$ & $<15 \mathrm{~Gy}$ & 15-15.5 Gy & $>15.5 \mathrm{~Gy}$ \\
\hline Rectal Mucosa V14 Gy & $<0.5 c c$ & $0.5-1 c c$ & $>1 c c$ \\
\hline Rectum Posterior Wall & $<8.5 \mathrm{~Gy}$ & $8.5-9.5 \mathrm{~Gy}$ & $>9.5 \mathrm{~Gy}$ \\
\hline Bladder Dmax to $0.1 \mathrm{cc}$ & $<110 \%$ TD & 110-120\% TD & $>120 \% \mathrm{TD}$ \\
\hline Bladder V19 Gy & $<10 c c$ & $10-15 c c$ & $>15 c c$ \\
\hline Bladder V17 Gy & $<15 \%$ & $15-20 \%$ & $>20 \%$ \\
\hline Bladder V9 Gy & $<50 \%$ & $50-60 \%$ & $>60 \%$ \\
\hline Urethra PRV Dmax to $0.1 \mathrm{cc}$ & $<110 \%$ TD & $110-115 \%$ TD & $>115 \% \mathrm{TD}$ \\
\hline Urethra PRV V105\% TD & $<5 \%$ & $5-15 \%$ & $>15 \%$ \\
\hline Neck of Femurs Dmax to $0.1 \mathrm{cc}$ & $<8 \mathrm{~Gy}$ & 8-9 Gy & 9 Gy \\
\hline Penile Bulb Dmax to 0.1cc (Recommended) & $100 \%$ TD & $100-105 \%$ TD & $>105 \% \mathrm{TD}$ \\
\hline Penile Bulb V10 Gy (Recommended) & $<3 c c$ & $3-5 c c$ & $>5 c c$ \\
\hline Intermediate Dose Spillage: ratio of volumes receiving 50\% TD to 100\% TD & $<4$ & $4-5$ & $>5$ \\
\hline Total Monitor Units & $<3 \times$ Dose in cGy & $3-3.5 \times$ Dose in cGy & $>3.5 \times$ Dose in cGy \\
\hline High Dose Conformation: Volume receiving $100 \%$ of TD divided by volume of PTVsbrt & $<1.1$ & $1.1-1.2$ & $>1.2$ \\
\hline
\end{tabular}

${ }^{a}$ Where Urethra has been limited to $19 \mathrm{~Gy}$, these volumes may exclude the urethra: eg. CTVsbrt D98 = CTVsbrt - Urethra PRV D98

either in the inferior-superior or anterior-posterior planes, only a lateral Electronic Portal Imaging (EPI) is necessary during treatment $[22,23]$.

\section{Data collection}

Patients will be assessed at baseline, the completion of treatment, 6 weeks post treatment, and then six monthly thereafter up to 5 years. At all visits, CTCAE GI and GU toxicity will be recorded. PSA will be measured at baseline, and then at all visits following treatment. The main efficacy endpoint is bNED calculated by the Phoenix definition of nadir+ 2 [24]. Any disease relapses or initiation of salvage treatments will also be recorded. Patient related quality of life (EPIC-26), and questionnaires regarding tolerance of treatment will also be collated at baseline, end of treatment, 12 months, 36 months and 60 months.

\section{Sample size}

The actuarial rates of CTCAE late grade 2-3 GI and GU toxicity will be calculated and reported at the 5 year mark. Studies using HDRB boost as well as IG-IMRT suggest rates of $5-15 \%$ are achievable. The study will be powered to recruit sufficient numbers to be confident

Table 3 Phase two EBRT component dose constraints

\begin{tabular}{llll}
\hline Structure & Per-Protocol & Minor Variation & Major Variation \\
\hline PTVebrt D95 & $>46$ Gy & $44-46$ Gy & $<44$ Gy \\
Prostatic Urethra PRV & Dmax < 47 Gy & Dmax: 47-50 Gy & Dmax > 50 Gy \\
Small Bowel & Dmax <47 Gy & Dmax: 47-50 Gy & Dmax $>50$ Gy \\
Neck of Femur & Dmax <35 Gy & Dmax: 35-45 Gy & Dmax $>45$ Gy \\
Rectum V45 & $<25 \%$ & $25-35 \%$ & $>35 \%$ \\
Bladder V45 & $<25 \%$ & $25-35 \%$ & $>35 \%$ \\
\hline
\end{tabular}




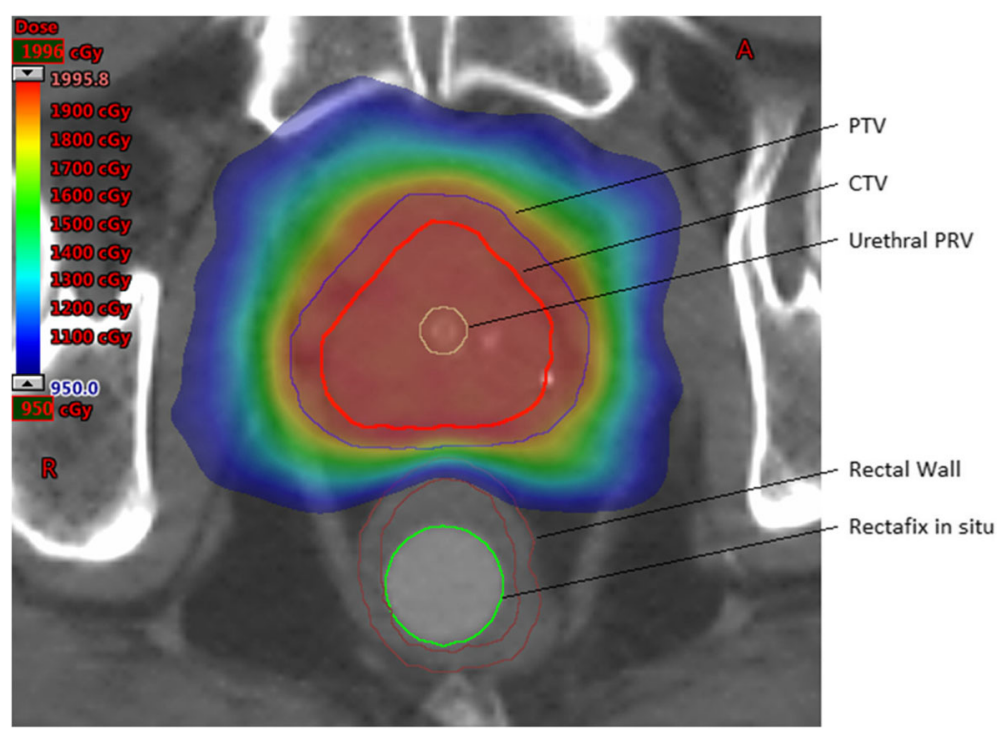

Fig. 2 Transverse CT view of example dose distribution adhering to planning constraints with Rectafix ${ }^{T M}$ displacing rectum posteriorly

that $<15 \%$ of men will have either a grade $2-3$ GI or grade 2-3 GU late GU event. The formula is:

$$
\mathrm{n}=(1.96 / \mathrm{E}) 2 \mathrm{p}(1-\mathrm{p})
$$

Where $\mathrm{E}$ is the margin of error. A conservative upper estimate of p here is 0.225 (the probability of an event). E might be 0.05 since that at worst gives a $95 \% \mathrm{CI}$ as $0.15+/-0.05$. With these figures $n=268$.

\section{Feasibility}

The protocol is deemed to be feasible if all of the following criteria are met:
- At least 3 different centres participate.

- Each centre accrues at least 5 patients.

- At least two centres attempt dose escalation.

\section{Discussion}

Several randomized controlled trials have demonstrated superior outcomes of a brachytherapy boost approach for intermediate and high risk PC compared with EBRT alone $[25,26]$. Despite this, the use of brachytherapy continues to regress [27]. Concurrently, the wider availability of new technology has created an opportunity to try to replicate brachytherapy boost type radiotherapy treatment plans which can be delivered using standard

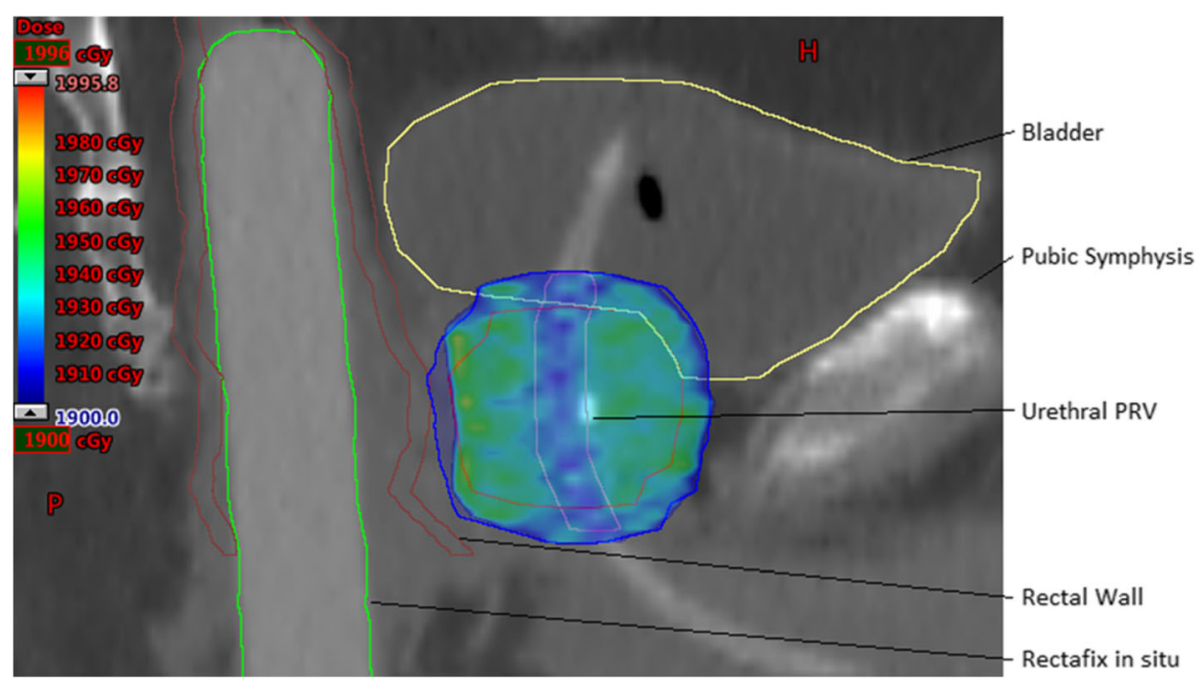

Fig. 3 Sagittal CT view of example dose distribution. Note the high dose sparing of the Urethral PRV, and posterior PTVsbrt dose compromise to meet rectal dose constraints 
EBRT equipment. The higher daily doses in the SBRT component are potentially very harmful to normal tissues, hence meticulous attention has been made in this study to minimise the risks to patients. Although three main endpoints are noted, it is the risk of severe toxicity which is prioritized in calculating the necessary patient numbers.

The management of such men is complicated by the integration of ADT, the investigation of newer agents, and the simultaneous investigation of SBRT monotherapy. The PROMETHEUS trial explores whether optimisation of the EBRT component is possible by translating the HDRB boost regimen into a non-invasive equivalent which can be more widely deployed. The structure of a future randomized trial is difficult to predict given the ongoing evolution in practice, however the PROMETHEUS schedule could be flexibly combined with various non-cytotoxic agents given the freedom to do so in the protocol.

\begin{abstract}
Abbreviations
ABR: Alpha/beta ratio; ADT: Androgen deprivation therapy; AE: Adverse event; ARTG: Australian register of therapeutic goods; bNED: Biological nonevidence of disease; CBCT: Cone beam computerised tomography; CK: Cyberknife; CT: Computerised tomography; CTC AE: Common terminology criteria for adverse events; CTV: Clinical target volume; DRE: Digital rectal examination; EBRT : External beam radiation therapy; ECE: Extracapsular extension; ECOG: Eastern Cooperative Oncology Group: EPI: Electronic portal imaging; Gl: Gastro intestinal; GU: Genito urinary; HDRB: High dose rate brachytherapy; ICRU: International commission of radiation units and measurements; IDC: In-dwelling urinary catheter; IGRT: Image guided radiation therapy; IMRT: Image guided radiation therapy; KV: Kilovoltage; LDRB: Low dose rate brachytherapy; MRI: Magnetic resonance imaging; MSKCC: Memorial sloan kettering cancer center; MV: Megavoltage; MVCT: Megavoltage computerised tomography; NOF: Neck of femur; OAR: Organ at risk; PC: Prostate cancer; PCSS: Prostate cancer specific survival; PRV: Planning target at risk volume; PSA: Prostate specific antigen; PTV: Planning target volume; QA: Quality assurance; RDD: Rectal displacement device; RT: Radiation therapy; RTOG: Radiation therapy oncology group; SBRT: Stereotactic body radiotherapy; SVI: Seminal vesicle invasion; VMAT: Volumetric modulated arc therapy
\end{abstract}

\section{Authors' contributions}

MR drafted the manuscript and revised it critically for important intellectual content; JM \& MS made substantial contributions to conception and design and revised the manuscript critically for important intellectual content; SG, MG, DP, JB, LW, SA, SK revised the manuscript critically for important intellectual content. All authors read and approved the final manuscript.

\section{Ethics approval and consent to participate}

Ethics approval for this study was granted by the South Western Sydney Local Health District Human Research Ethics Committee on the 2/12/2013 reference number HREC/13/LPOOL/311. Written informed consent was obtained from all participants.

\section{Competing interests}

The authors declare that they have no competing interests.

\section{Publisher's Note}

Springer Nature remains neutral with regard to jurisdictional claims in published maps and institutional affiliations.

\section{Author details}

${ }^{1}$ Calvary Mater Newcastle, Newcastle, NSW, Australia. ${ }^{2}$ Liverpool and Macarthur Cancer Therapy Centres, Sydney, NSW, Australia. Ingham Institute,
Liverpool, NSW, Australia. ${ }^{4}$ Princess Alexandra Hospital, Brisbane, QLD, Australia. ${ }^{5}$ St. George Hospital Cancer Centre, Sydney, NSW, Australia.

Received: 9 February 2017 Accepted: 16 May 2018

Published online: 24 May 2018

\section{References}

1. Dearnaley D, Syndikus I, Mossop H, Khoo V, Birtle A, Bloomfield D, et al. Conventional versus hypofractionated high-dose intensity-modulated radiotherapy for prostate cancer: 5-year outcomes of the randomised, noninferiority, phase 3 CHHiP trial. Lancet Oncol. 2016;17(8):1047-60.

2. Watkins Bruner D, Pugh SL, Lee WR, Dignam JJ, Low D, Swanson GP, et al. NRG oncology/RTOG 0415, phase 3 noninferiority study comparing 2 fractionation schedules in patients with low-risk prostate Cancer: prostatespecific quality of life results. Int J Radiat Oncol Biol Phys. 2016:96(2):S2-3.

3. Catton CN, Lukka H, Gu CS, et al. Randomized Trial of a Hypofractionated Radiation Regimen for the Treatment of Localized Prostate Cancer. J Clin Oncol. 2017:35:1884-90.

4. Hayden AJ, Martin JM, Kneebone AB, Lehman M, Wiltshire KL, Skala M, et al. Australian \& new Zealand Faculty of Radiation Oncology Genito-Urinary Group: 2010 consensus guidelines for definitive external beam radiotherapy for prostate carcinoma. J Med Imaging Radiat Oncol. 2010;54(6):513-25.

5. Proust-Lima C, Taylor JM, Secher S, Sandler H, Kestin L, Pickles T, et al. Confirmation of a low alpha/beta ratio for prostate cancer treated by external beam radiation therapy alone using a post-treatment repeatedmeasures model for PSA dynamics. Int J Radiat Oncol Biol Phys. 2011; 79(1):195-201

6. Kuban DA, Levy LB, Cheung MR, Lee AK, Choi S, Frank S, et al. Long-term failure patterns and survival in a randomized dose-escalation trial for prostate cancer. Who dies of disease? Int J Radiat Oncol Biol Phys. 2011; 79(5):1310-7.

7. Viani GA, da Silva LG, Stefano EJ. High-dose conformal radiotherapy reduces prostate cancer-specific mortality: results of a meta-analysis. Int J Radiat Oncol Biol Phys. 2012;83(5):e619-25.

8. Pollack A, Zagars GK, Starkschall G, Antolak JA, Lee J, Huang E, et al. Prostate cancer radiation dose response: results of the M. D. Anderson phase III randomized trial. Int J Radiat Oncol Biol Phys. 2002:53(5):1097-105.

9. Khor R, Duchesne G, Tai KH, Foroudi F, Chander S, Van Dyk S, et al. Direct 2arm comparison shows benefit of high-dose-rate brachytherapy boost vs external beam radiation therapy alone for prostate cancer. Int J Radiat Oncol Biol Phys. 2013;85(3):679-85.

10. Zwahlen DR, Andrianopoulos N, Matheson B, Duchesne GM, Millar JL. High-dose-rate brachytherapy in combination with conformal external beam radiotherapy in the treatment of prostate cancer. Brachytherapy. 2010;9(1):27-35.

11. Martin JM, Bayley A, Bristow R, et al. Image guided dose escalated prostate radiotherapy: still room to improve. Radiat Oncol 2009;4:50

12. Martin JM, Frantzis J, Eade T, Chung P. Clinician's guide to prostate IMRT plan assessment and optimisation. J Med Imaging Radiat Oncol. 2010;54(6):569-75.

13. Kupelian PA, Willoughby TR, Reddy CA, Klein EA, Mahadevan A. Hypofractionated intensity-modulated radiotherapy (70 Gy at 2.5 Gy per fraction) for localized prostate cancer: Cleveland Clinic experience. Int J Radiat Oncol Biol Phys. 2007;68(5):1424-30.

14. Cahlon O, Zelefsky MJ, Shippy A, Chan H, Fuks Z, Yamada Y, et al. Ultra-high dose (86.4 Gy) IMRT for localized prostate cancer: toxicity and biochemical outcomes. Int J Radiat Oncol Biol Phys. 2008;71(2):330-7.

15. Spratt DE, Zumsteg ZS, Ghadjar P, Kollmeier MA, Pei X, Cohen G, et al. Comparison of high-dose (86.4 Gy) IMRT vs combined brachytherapy plus IMRT for intermediate-risk prostate cancer. BJU Int. 2014;114(3):360-7.

16. Isacsson U, Nilsson K, Asplund S, Morhed E, Montelius A, Turesson I. A method to separate the rectum from the prostate during proton beam radiotherapy of prostate cancer patients. Acta Oncol. 2010;49(4):500-5.

17. Song DY, Herfarth KK, Uhl M, Eble MJ, Pinkawa M, van Triest B, et al. A multi-institutional clinical trial of rectal dose reduction via injected polyethylene-glycol hydrogel during intensity modulated radiation therapy for prostate cancer: analysis of dosimetric outcomes. Int J Radiat Oncol Biol Phys. 2013;87(1):81-87.

18. Heidenreich A, Bellmunt J, Bolla M, Joniau S, Mason M, Matveev V, et al. EAU guidelines on prostate cancer. Part 1: screening, diagnosis, and treatment of clinically localised disease. Eur Urol. 2011;59(1):61-71. 
19. King BL, Butler WM, Merrick GS, Kurko BS, Reed JL, Murray BC, et al. Electromagnetic transponders indicate prostate size increase followed by decrease during the course of external beam radiation therapy. Int J Radiat Oncol Biol Phys. 2011;79(5):1350-7.

20. Martin JM, Brett R, Blyth J, Morrison S, Bryant D, Plank A, et al. Dosimetric effect of external beam planning preceding combined high-dose-rate brachytherapy of the prostate. Brachytherapy. 2011;10(6):474-8.

21. Curtis W, Khan M, Magnelli A, Stephans K, Tendulkar R, Xia P. Relationship of imaging frequency and planning margin to account for intrafraction prostate motion: analysis based on real-time monitoring data. Int J Radiat Oncol Biol Phys. 2013;85(3):700-6.

22. Kindblom J, Ekelund-Olvenmark AM, Syren H, lustin R, Braide K, FrankLissbrant I, et al. High precision transponder localization using a novel electromagnetic positioning system in patients with localized prostate cancer. Radiother Oncol. 2009:90(3):307-11.

23. Litzenberg DW, Balter JM, Hadley SW, Sandler HM, Willoughby TR, Kupelian $\mathrm{PA}$, et al. Influence of intrafraction motion on margins for prostate radiotherapy. Int J Radiat Oncol Biol Phys. 2006;65(2):548-53.

24. Roach M 3rd, Hanks G, Thames H Jr, Schellhammer P, Shipley WU, Sokol GH, et al. Defining biochemical failure following radiotherapy with or without hormonal therapy in men with clinically localized prostate cancer: recommendations of the RTOG-ASTRO Phoenix consensus conference. Int J Radiat Oncol Biol Phys. 2006;65(4):965-74.

25. Sathya JR, Davis IR, Julian JA, Guo Q, Daya D, Dayes IS, et al. Randomized trial comparing iridium implant plus external-beam radiation therapy with external-beam radiation therapy alone in node-negative locally advanced cancer of the prostate. J Clin Oncol. 2005;23(6):1192-9.

26. Morris WJ, Tyldesley S, Rodda S, et al. Androgen Suppression Combined with Elective Nodal and Dose Escalated Radiation Therapy (the ASCENDE-RT Trial): An Analysis of Survival Endpoints for a Randomized Trial Comparing a Low-Dose-Rate Brachytherapy Boost to a Dose-Escalated External Beam Boost for High-and Intermediate-risk Prostate Cancer. Int J Radiat Oncol Biol Phys. 2017;98:275-85.

27. Petereit DG, Frank SJ, Viswanathan AN, Erickson B, Eifel P, Nguyen PL, et al. Brachytherapy: where has it gone? J Clin Oncol. 2015;33(9):980-2.

\section{Ready to submit your research? Choose BMC and benefit from:}

- fast, convenient online submission

- thorough peer review by experienced researchers in your field

- rapid publication on acceptance

- support for research data, including large and complex data types

- gold Open Access which fosters wider collaboration and increased citations - maximum visibility for your research: over $100 \mathrm{M}$ website views per year 